\title{
Efficient Table-Based Polynomial on FPGA
}

\author{
Marco Barbone*, Bas W. Kwaadgras ${ }^{\dagger}$, Uwe Oelfke $^{\ddagger}$, Wayne Luk* and Georgi Gaydadjiev ${ }^{\S *}$ \\ ${ }^{*}$ Department of Computing, Imperial College London, London, UK \\ ${ }^{\S}$ Bernoulli Institute, University of Groningen, Groningen, Netherlands \\ Email: m.barbone19@imperial.ac.uk,w.luk@imperial.ac.uk, g.gaydadjiev@rug.nl \\ $\ddagger$ Joint Department of Physics at The Institute of Cancer Research and The Royal Marsden NHS Foundation Trust, \\ London, UK \\ ${ }^{\dagger}$ Maxeler Technologies, London, UK
}

\begin{abstract}
Field Programmable Gate Arrays (FPGAs) are gaining popularity in the context of scientific computing due to the recent advances of High-Level Synthesis (HLS) toolchains for customised hardware implementations combined with the increase in computing capabilities of modern FPGAs. As a result, developers are able to implement more complex scientific workloads which often require the evaluation of univariate numerical functions. In this study, we propose a methodology for tablebased polynomial interpolation aiming at producing area-efficient implementations of such functions on FPGAs achieving the same accuracy and at similar performance as direct implementations. We also provide a rigorous error analysis to guarantee the correctness of the results. Our methodology covers the forecast of resource utilisation of the polynomial interpolator and, based on the characteristics of the function, guides the developer to the most area-efficient FPGA implementation. Our experiments show that in the case of a radiation spectrum of a Black Body application based on evaluating Planck's Law, it is possible to reduce resource utilisation by up to $90 \%$ when compared to direct implementations not using table-based methods. Moreover, when only the kernels are considered, our method uses up to two orders of magnitude fewer resources with no performance penalties. Based on previous more theoretical works, our study investigates practical applications of table-based methods in the context of high performance and scientific computing where it is used to implement common but more complex functions than the elementary functions widely studied in the related literature.
\end{abstract}

\section{INTRODUCTION}

Recently, Field Programmable Gate Arrays (FPGAs) became very popular as accelerators for scientific applications. This is mainly powered by the recent advances of HighLevel Synthesis (HLS) toolchains for customised hardware implementations [1]-[4] along with the continuous scaling of resource capacities and speeds of modern FPGA devices. As a consequence, larger scale applications have been implemented on systems incorporating FPGA accelerators. For example, CERN is investigating the possibility to port its High Energy Physics (HEP) workflows to FPGAs [5]. Many scientific computing applications require the evaluation of real functions $f(x)$ of one real variable $x$; typically elementary functions, such as trigonometric functions, logarithms and square roots. However, when a compound function (for example $\left.\cos \left(\sin \left(7 x^{5}\right)\right)\right)$ is required, it is usually more area-efficient to implement the latter as one single operator instead of a combination of basic operators [6]. Specific methods exist for implementing the majority of elementary functions; for example, the CORDIC algorithm and its variations [7] can be used to design specific and optimised implementations for most common compound functions. However, implementing each compound function would require a lot of non-reusable work. As a result, this approach does not scale; large scale applications especially in the context of scientific computing might require a large number of compound functions of practical complexity, as in the case of Jacobi Elliptic Functions (JEFs), which will be discussed in this paper. Moreover, the CORDIC derivative results in iterative small operators but are less suitable for both high throughput or low latency applications due to their multi-cycle execution delays [6], [8].

A more general method is to store all possible values of the function output in a lookup table. This method, however, is limited to only small data types since the table size increases exponentially with the bitwidth of the input data type. Instead of storing all output values, one can greatly reduce the table size by storing only a sub-set of the values and apply a suitable interpolation function, hence trading on-chip memory resources for logic and/or the specialised multipliers present on FPGAs known as DSPs.

Extensive literature describing table-based polynomials exists. However, most focus on elementary functions [9], optimise the degree of the polynomial [10], support only up to 24 bits of precision [8], and use only fixed-point or only floatingpoint but not both data types [11], [12]. We consider these studies more on the theoretical side as they mainly focus on elementary functions whose highly optimised implementations are both available open-source [13] and are available in most vendor tools, so users are more likely to use ready-to-use implementations than to re-implement them using table-based methods. In this study, we do a practical investigation of the use of table-based methods in the context of high performance and scientific computing targeting popular functions that are less likely to be available open-source or present in vendor tools. Furthermore, implementations provided in previous studies are either in lower-level languages such as VHDL and Verilog or the VHDL is generated from a higherlevel language such as $\mathrm{C}$ or $\mathrm{C}++$. The advantage of using VHDL and Verilog is the fine-grain control of every part of the design allowing both optimisations and easy integration and reuse in large projects. On the other hand, it is challenging 
or often impossible to integrate such frameworks in HighLevel Synthesis (HLS) toolchains. Many HLS toolchains do not even support VHDL and Verilog integration. In the case of generated Verilog and VHDL, any change in the application would require a re-generation and a re-integration of the code which can result in a lot of engineering effort. Moreover, many optimisations are not portable as they depend on the targeted FPGA characteristics. When a different FPGA is targeted, such optimisations can lead to increased resource utilisation and even clock frequency reduction, when compared to an unoptimised, generic implementation, not tied to any particular FPGA. However, when the aim is to achieve the lowest possible resource utilisation while targeting a specific FPGA, most likely, HLS toolchains will not be used because they do not allow low-level control over the design. We emphasise that instead, our study aims at a tool for minimising the engineering effort while significantly saving resources, resulting in practical, reasonably optimised FPGA accelerated applications.

To achieve higher portability, our study presents a generalpurpose methodology not relying on any hardware-specific optimisation. Our methodology exposes selected parameters that can be tuned to explore trade-offs between different hardware resources. These trade-offs are carefully explained using Xilinx's VU9P FPGA as a motivational example of how to apply our methodology. Furthermore, the methodology allows users to quickly estimate the resources needed for a table-based implementation, and decide early (before writing any line of code) whether it will be more resourceefficient than a direct implementation. Moreover, the proposed methodology is generally applicable, supports both fixed-point and floating-point data types and has no upper bound in terms of achievable precision. We evaluate our methodology using the Cubic Spline polynomial interpolation, which requires very little engineering effort to implement and is therefore practical in many applications, especially in the context of scientific computing where table-based methods can be used in different portions of the application. We show that, when implementing Cubic Spline-based functions, it is possible to save several orders of magnitude of resources with limited engineering effort. While more advanced table-based methods, may lead to lower resource utilisation, the amounts saved stay in the same order of magnitude as when using Cubic Spline, after investing significantly more engineering effort. Compared to the above-mentioned methods, our methodology based on Cubic Splines has two major limitations: first, it requires sampling both the function and its derivative, second, it uses only a degree three polynomial interpolator. More involved methods require sampling the function only (as opposed to both the function and its derivative), thus at least halving the memory requirements to achieve the same accuracy. These methods might further reduce the memory utilisation since they are inherently more accurate; they can achieve the same error as Cubic Splines interpolation while sampling fewer points. This reduction in sampling point can be translated into fewer arithmetic operations since involved methods allow to tune the degree of the polynomial interpolator. A lower polynomial degree will decrease the number of arithmetic operations but increase the number of sample points. When a more area-efficient method than Cubic Spline is required, the users can still use our methodology and derive an approximate upper bound for the expected resource utilisation of their preferred table-based method.

For the sake of generality, we present all necessary implementation details as it is practically impossible to provide an API for every HLS toolchain, currently existing or yet to be proposed. Instead, we implemented our methodology in MaxJ and Maxeler Technologies Ltd integrated it into their MaxPower library starting from version 2020.2. MaxPower's source code is accessible to academic users via the Maxeler University Program (MAX-UP). As a result, any MaxJ developer can use our API in their applications, benefit from and further optimise our implementation. Naturally, this allows easy reproduction of the results presented here.

The main contributions of this study are:

- Generic methodology for resource estimation and a formal error analysis that derives a table-based architecture delivering accurate results with no upper precision limit;

- Support for both floating- and fixed-point data types optimising the internal logic to avoid unneeded computation;

- Up to $90 \%$ reduced resource usage compared to the direct method for representative numerical problems in scientific computing, including challenging physics simulation.

The remainder of this paper is organised as follows. Section II discusses related concepts from physics and mathematics. Section III introduces our methodology, explaining in detail the error analysis and the resources estimation. Section IV presents the validation methodology and the achieved resource utilisation based on three use cases. Finally, Section V concludes the paper and indicates promising future directions.

\section{BACKGROUND}

This section explains the fundamental knowledge required to contextualise the use cases used in our experiments.

\section{A. Jacobi elliptic functions}

The Jacobi elliptic functions (JEFs) [14] are standard forms of elliptic functions. They derive from the inversion of the elliptic integral of the first kind

$$
u=F(\phi, k)=\int_{0}^{\phi} \frac{d t}{\sqrt{1-k^{2} \sin ^{2} t}},
$$

with $0<k^{2}<1$, where $k$ is known as the elliptic modulus, and $\phi=F^{-1}(u, k)=a m(u, k)$ is the Jacobi amplitude. The three basic JEFs $c n(u, k), d n(u, k)$ and $s n(u, k)$ follow from Equation 1 and are defined as:

$$
\begin{aligned}
& \operatorname{sn}(u, k)=\sin \phi=\sin (\operatorname{am}(u, k)) \\
& c n(u, k)=\cos \phi=\cos (\operatorname{am}(u, k)) \\
& d n(u, k)=\sqrt{1-k^{2} \sin ^{2} \phi}=\sqrt{1-k^{2} \sin ^{2}(a m(u, k))} .
\end{aligned}
$$


In-depth discussions about the definition of JEFs are out of the scope of this study; Bowman [15] provides more details about their theory and applications. The JEFs appear in several physics problems and the conformal mapping of engineering problems [15]-[18]. The JEFs provide a complete description or a proper approximation of a generalisation of the classic pendulum problem [19]. Given the diversity and the number of physics problems where JEFs appear, we will use them to benchmark the efficiency of our approach for such physics and engineering applications. For our experiments, out of the three basic JEFs, we considered the Jacobi SN function which is a particularly good candidate for table-based polynomial interpolation since it is periodic and computing it using the common mean method requires a lot of hardware resources.

\section{B. Legendre polynomials}

The Legendre polynomial $P_{n}(x)$ can be defined by the contour integral

$$
P_{n}(x)=\frac{1}{2 \pi i} \oint \sqrt{1-2 t x+t^{2}} t^{-n-1} d t,
$$

where the contour encloses the origin and is traversed in a counterclockwise direction [20]. The Legendre polynomials are commonly used to treat many problems both in physics and mathematics. For example, these polynomials can be used to derive many identities useful in quantum mechanics, electromagnetism and optics [21]. The associated forms appear in the definition of the spherical harmonics, thus playing a vital role in finding solutions to a range of differential equations (for example, the Laplace equation) in spherical coordinates [22]. Recently, Voelker et al. [23] proposed the Legendre Memory Unit, a novel memory for recurrent neural networks based on the Legendre polynomials, that dynamically maintains information across long windows of time using relatively few resources. Legendre polynomial can be of any degree; in this study we consider Legendre polynomial of degree 10.

\section{Black-Body radiation}

Black-body radiation is the thermal electromagnetic radiation within or surrounding a body in thermodynamic equilibrium with its environment, emitted by a black-body, an idealised non-reflective body. It has a specific spectrum of wavelengths, inversely related to intensity, that depends only on the body's temperature, which is assumed uniform and constant for the sake of simplicity [24]. Finding an analytical expression for black-body spectral radiance that matches experimental results was a major problem in theoretical physics, which was solved by Max Planck with the law of blackbody radiation [25]. Attempts to develop a physical theory that would produce Planck's law then led to the PlanckEinstein relation, which was a major step in the development of Quantum Physics [26]. Planck's law states that [27]:

$$
B_{\lambda}(\lambda, T)=\frac{2 h c^{2}}{\lambda^{5}} \frac{1}{e^{h c /\left(\lambda k_{B} T\right)}-1}
$$

where:
- $B_{\lambda}(\lambda, T)$ is the spectral radiance density of radiation of wavelength $\lambda$, per unit wavelength, at thermal equilibrium at temperature $T$.

- $h$ is the Planck constant;

- $c$ is the speed of light in a vacuum;

- $k$ is the Boltzmann constant;

- $\lambda$ is the wavelength of the electromagnetic radiation;

- $T$ is the absolute temperature of the body.

Black-bodies are commonly used in many different applications, for example, in lighting, heating, security, thermal imaging and testing and measurement applications. Since the intensity of the energy can be determined using the Planck Law of radiation, a black-body radiation source with a known or at least measurable temperature, is used for calibrating and testing radiation thermometers. In contrast to the JEFs and Legendre polynomials, Planck's law solves a more practical problem. It is used here as a representative of a simple physics application that can greatly benefit from our approach.

\section{Method}

Given are a function $f: I \rightarrow \mathbb{R}$ (where $I=[0,1)$ ) and its approximation $\tilde{f}$. Since computer arithmetic precision is limited we claim that $f(x)=\tilde{f}(x)$ if the interpolation error of $\tilde{f}(x)$ is smaller than computer arithmetic precision. While the values of $x$ are limited by $x \in[0,1)$, there is no such guarantee for the values of $f(x)$, so using the relative error to measure the difference between $f(x)$ and $\tilde{f}(x)$ is not appropriate for every $x$ as $f(x)$ might be a either a small or a large number. Equation 5 from Ericson [28] provides a combined relative and absolute error metric that is consistent for all $x$ independent of their value. This equation assumes that the same error margin is used for both relative and absolute error.

$$
\epsilon(x) \leq \delta * \max (1,|f(x)|,|\tilde{f}(x)|)
$$

where:

- $\epsilon(x)=|f(x)-\tilde{f}(x)|$;

- $\delta$ is the tolerated error margin.

To guarantee that $f(x)$ and $\tilde{f}(x)$ are equivalent, we set $\delta=u l p(x)^{1}$ or $\delta=\frac{1}{2} u l p(x)$ (if rounding to nearest even is used) and require Equation 5 to be satisfied for all $x$. If it is, then we can claim that $f(x)=\tilde{f}(x)$. Hence, without loss of precision, $f(x)$ can be evaluated by computing $\tilde{f}(x)$. Given the flexibility offered by FPGAs, it is always possible to choose a data type that utilises the minimum number of bits to represent the range of values needed to solve the problem by respecting the accuracy constraints. Thus, we assume that the accuracy is $\frac{1}{2} u l p(x)$ in all cases. In case more or less accuracy is required, the user can adjust the data types.

In this study, $\tilde{f}(x)$ is computed using piece-wise polynomial interpolation. We have chosen Cubic Spline as the interpolation method, where the values are interpolated by using a third-order polynomial [29]. While other interpolation methods, such as least-squares approximation [30], minimax

\footnotetext{
${ }^{1}$ Unit in the last place (ulp) is the spacing between two consecutive floatingpoint numbers.
} 
computed by using the Remez algorithm [31] and rational approximations [32], can achieve the required accuracy with smaller tables and offer more flexibility (by allowing the use of polynomials of any order), as mentioned in Section I, their implementation requires more engineering effort. The implementation of Cubic Spline is simpler and the error estimation can be computed using Equation 6 as will be discussed in Section III-B. The simplicity of Cubic Spline facilitates the adoption of our methodology in the context of scientific computing, where saving orders of magnitude of resources is more important than saving a few extra DSPs or LUTs as it can make the difference between a design that fits or not on the target FPGA. Moreover, Cubic Spline allows for testing our methodology in a worst-case scenario in which one of the least area-efficient polynomial interpolators can still be better than the respective direct method. However, computing $\tilde{f}(x)$ instead of $f(x)$ is advantageous only if the hardware resources needed by $\tilde{f}(x)$ are fewer than computing $f(x)$. Since the order of the polynomial is fixed in this case the only variable left is the size of the Polynomial Look Up Table (P-LUT) to store all the sampling points. Using Equation 7 one can easily determine the P-LUT size which can be used to estimate the costs at compile time ahead of hardware synthesis. Section III-C will provide more details on the resource estimation.

Figure 1 shows the proposed methodology. First, the user identifies suitable numerical functions of one variable. The user also chooses a cut-off that represents the maximum size of the P-LUT; this cut-off would usually depend on available onchip memory resources and the target clock frequency (since large monolithic memories tend to be harder to place and route). Then, using Equation 7, the P-LUT size is estimated. In case the size is lower than the cut-off, the function can be implemented using a table-based polynomial interpolation; otherwise, it is not suitable and other strategies, such as a direct implementation, should be used. If the function is suitable for table-based polynomial implementation, in the case of low precision, it is possible to further optimise the implementation. When the P-LUT size is lower than a certain limit, it is possible to sample all the values instead of performing a polynomial interpolation, which saves the DSPs required for the multiplications. This limit is platform-dependent and, in this study, is set to the size of one Block RAM (BRAM). The next step is type optimisation. At this point, all the sampled values are available, so in the case where the input data type is a fixed-point, it is possible to compute the minimum $(v)$ and the maximum $(V)$ value. With $R=V-v$ it is possible to tune the data type such that $R$ is represented using the minimum number of bits without any precision loss. Finally, the P-LUT is built, the Cubic Spline interpolator is instantiated if required, and the resulting VHDL is generated.

\section{A. Polynomial Look Up Table}

P-LUTs are used to store the sampling points needed by the piece-wise polynomial interpolation. Since the proposed method aims to support both floating-point and fixed-point

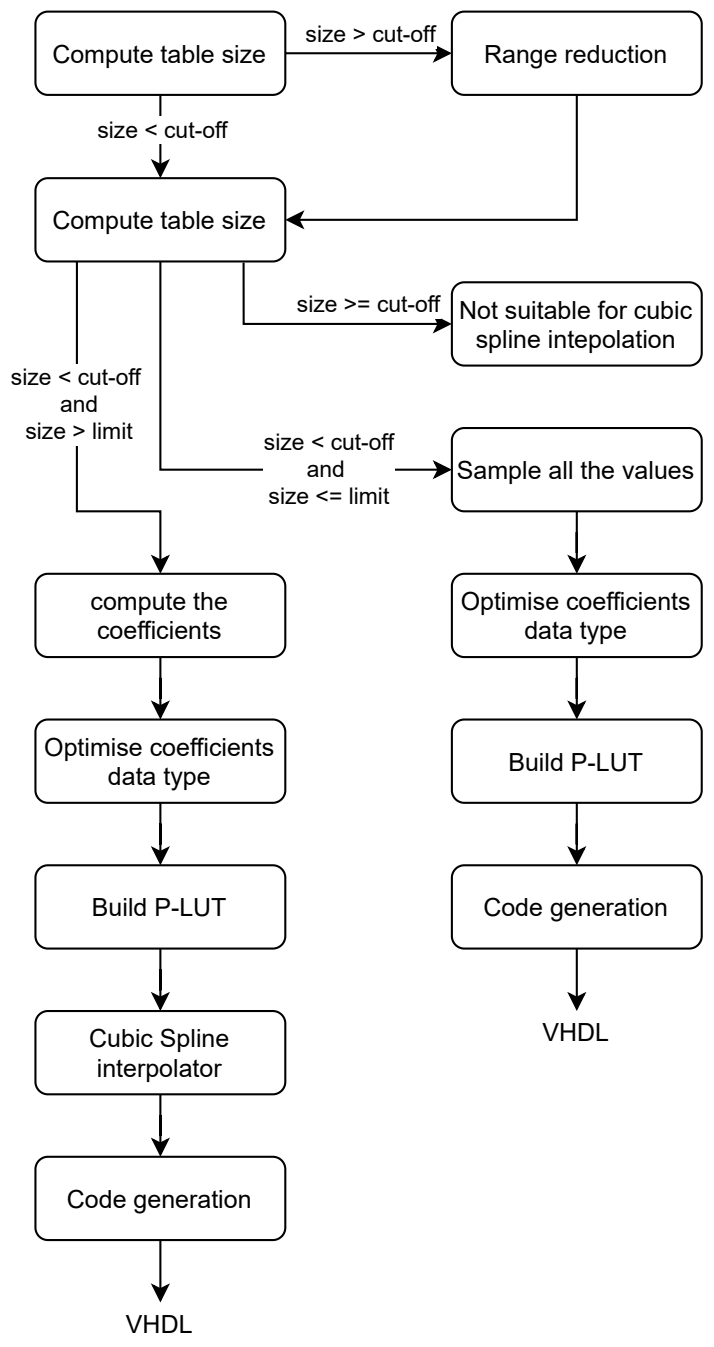

Figure 1. Methodology used to build the operator

data types, there are two P-LUT implementations. As mentioned in Section III, the design requirement is $I=[0,1)$. This does not cause any loss of generality since any finite generic interval $[a, b)$ can be translated and scaled into $[0,1)$ using a range reduction algorithm. In-depth discussions about range reduction are out of the scope of this study but Muller [7] provides different viable strategies for most of the elementary functions. This constraint simplifies the design of the PLUT and allows to save hardware resources. For the sake of simplicity, the depth of the P-LUT is forced to be a power of two by rounding up to the next power of two the result of Equation 6. While rounding up can almost double the P-LUT size in the worst case, it greatly simplifies the addressing logic and allows to fully utilise the address bits.

1) Fixed-point: The fixed-point data type consists of an integer and a fractional part in the form $i_{1} i_{2} \ldots i_{n} . f_{1} f_{2} \ldots f_{m}$. Since $f(x)$ is evaluated in the interval $I=[0,1), x$ is in the form $0 . f_{1} f_{2} \ldots f_{m}$ with the integer part containing no bits. Additionally, since the size of the P-LUT is a power of two (by design) the fractional part could be divided in 
$f_{1} f_{2} \ldots f_{w} f_{w+1} \ldots f_{m}$ where $w$ is the base-two logarithm of the P-LUT depth. The first $w$ bits directly address the memory location while the remaining $m-w$ bits are used to compute the Cubic Spline interpolation. This design allows to fully exploit the asymmetric structure of DSPs commonly used on current FPGAs, performing an asymmetric multiplication between $m-w$ and $m$ bits. For example, the VU9P FPGA, used in this study, supports $18 \times 27$ bits asymmetric DSP slices.

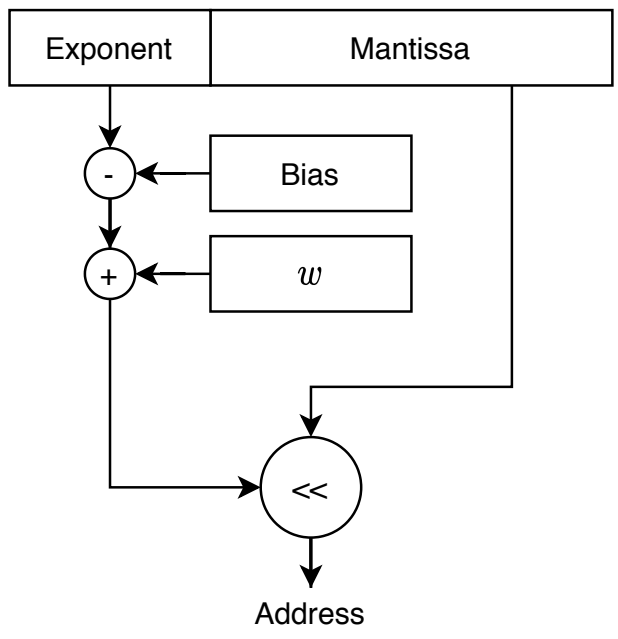

Figure 2. Floating-point address converter

2) Floating-point: The IEEE-754 floating-points are in the form of $1 . x_{1} x_{2} \ldots x_{n}$ multiplied with an integer power of 2 [33]. Please note that denormalized floating-points are not supported. As pointed out by Xilinx, since it is possible to specify the number of bits of the exponent, using denormalisation to extend the dynamic range is less resource-efficient than increasing the exponent size by one bit [34]. Differently from fixed-point representation, it is not possible to partition floating-points to directly address a P-LUT. Since $f(x)$ is evaluated in the interval $I=[0,1)$ the easiest way to determine the address from $x$ is to multiply $x$ by the size of the table and then cast the result to an integer using truncation rounding mode. This approach however has a high cost in terms of hardware resource utilisation, since it requires a floatingpoint multiplier and float to integer caster. Given the interval $I=[0,1)$ and the size of the P-LUT being a power of two, an optimised way to transform $x$ into an address that does not require multipliers and casters is:

1) Align the mantissa for an exponent of $w$ bits, where $2^{w}$ is equal to the depth of the P-LUT;

2) Slice the $w$ most significant bits as the address.

We stress that this method is used to compute only the address of the table; using the resulting integer in the numeric computation will cause a loss of precision. Since the slice operator does not require hardware resources, the cost is given by the normalisation. This normalisation only requires an integer addition of the exponent and $w$-bias followed by a left-shift of $m$, where $m$ is the number of the mantissa bits.
Since $w$ and bias are constants the subtraction $w$-bias is precomputed at compile-time. Hence, it does not require hardware resources.

\section{B. Error analysis}

Since $f$ is approximated with Cubic Spline, the approximation error of this particular interpolation is a very well-known problem that has already been solved in the literature. In particular, Halls [35] provides a well-known formula to quantify the approximation error of Cubic Spline. Theorem 1 in [35] states the following. Given $C^{k}(\Omega)$ the space of functions on $\Omega$ to $R$ that are $k$ times continuously differentiable [36]. Let $s$ be the Cubic Spline associated with $f \in C^{4}[a, b]$ and the partitioning $\pi$. Then

$$
\epsilon=\left\|s^{(r)}-f^{(r)}\right\| \leq \epsilon_{r}\left\|f^{(4)}\right\| h^{(4-r)}
$$

Where:

$$
\begin{aligned}
& \text { - } r=0 ;^{2} \\
& \text { - } \epsilon_{0}=5 / 384 ;^{3} \\
& \text { - } \pi: a=x_{0}<x_{1}<\ldots<x_{n}=b ; \\
& \text { - } h=\max _{i}\left\{x_{i}-x_{i-1}\right\} ;^{4} \\
& \text { - }\left\|f^{(4)}\right\|=\max \left(f^{(4)}\right) \text { in }[a, b] ;
\end{aligned}
$$

Equation 6 shows that the error depends on the function approximated and the sampling interval $h$. Equation 7 helps obtaining a sampling interval leading to interpolation error $<\epsilon_{\max }$.

$$
h=\sqrt[4]{\frac{\epsilon_{0} \epsilon_{\max }}{\left\|f^{(4)}\right\|}}
$$

The maximum error $\left(\epsilon_{\max }\right)$ is determined from the input type of the given function $f$. If the error is reduced to a value smaller than the smallest representable value of the given data type, the resulting interpolation error of the Cubic Spline is considered 0. In particular, in case of fixed-point representations, $\epsilon_{\max }=2^{-\left(f_{b}+1\right)}$, where $f_{b}$ is the number of fractional bits of the data type. Otherwise, for floating-points, assuming to near-even rounding is used, $\epsilon_{\max }=\frac{1}{2} u l p(x)$.

The rounding error is computed differently depending on the data type used. Users who need faithful rounding can extend the input type by $g$ guard bits [6], such that the architecture carries out all computation in the extended data type and round the result to the desired type. For example, Table I shows that there is no difference in the resource utilisation between 4,13 and 4,23 fixed-point data types; as a consequence, users requiring faithful rounding can extend the smaller input data type at little or no extra hardware cost. In the floating-point case, all the computations are carried out in floating-point. In this care the IEEE-754 standard already specifies guard bits and the arithmetic [33]. Thus, the only potential source of error is eventual cancellation or an ill-conditioned problem, both of which will affect the precision of the direct method

\footnotetext{
${ }^{2}$ Since $f \in C^{4}[a, b]$ then $r=0,1,2,3$, but to estimate the approximation error of $f$ only $r=0$ is used. The others values of $r$ are used to compute the approximation error of the first, second and third derivative of $f$.

${ }^{3}$ This term is part of the theorem.

${ }^{4}$ Please note that the sampling interval is uniform in this implementation.
} 
and the interpolation in the same way. The user concerned by the rounding error in the polynomial evaluation can implement one of the stable evaluation schemes proposed in [37].

\section{Resource estimation}

The proposed methodology performs a third-degree polynomial interpolation and a read from a P-LUT, which accounts for most of the resources needed. From Equation 7, the number of sampling points is $\frac{b-a}{h}$ but, since $b=1$ and $a=0$ by design choice, in our case the number of sampling points is simply $1 / h$. Cubic Splines store four coefficients for each sampling point, so the size of the P-LUT is 4 times the number of sampling points. By multiplying the number of coefficients with the bitwidth of the data type used, it is possible to obtain the P-LUT size in bits. Depending on the resources available on the FPGA, for example, BRAMs in the Xilinx VU9P FPGA device, the number of memory resources needed can be derived from the P-LUT size in bits. The polynomial interpolation consists of three additions and three multiplications. The logic resource utilisation varies greatly between fixed-points and floating-points. In the fixed-point case, most of the resources are used to perform the multiplication, while the additions consume a negligible number of LUTs and FFs, especially when the DSP slices contains adders. The exact number of DSPs utilised depends on the type of DSPs available; for example, in the Xilinx VU9P FPGA used in this study, the DSPs contain $18 \times 27$ multipliers. In this case, when fixed-point with four integer bits and 23 fractional bits is used, assuming that the P-LUT contains 128 elements, the interpolation computes three $16 \times 23$ multiplications, which requires only three DSPs. In the case of floating-points, the resource utilisation of additions is not negligible. The resource utilisation, in this case, depends both on the type of DSPs available on the FPGA and the floating-point operator utilised. In our study we utilised the Xilinx floating-point operator [34] and we left the parameters to their default values. Changing these parameters greatly influences the number of DSPs, LUTs and FFs required. In the case of commonly utilised floatingpoint data types, the amount of resources needed for this operator is reported in the official documentation [38]; in the other cases, it could be measured by developing microbenchmarks and instantiating the operators. For example, to implement a Cubic Spline interpolator that supports floatingpoints consisting of 8 exponent bits and 24 mantissa bits on the VU9P, it is necessary to instantiate an accumulator [39] that needs two DSPs and three multipliers that require two DSPs each, for a total of eight DSPs utilised.

\section{REsults}

We used MaxCompiler version 2020.2 and Vivado 2019.1 in our experiments. For fair comparison we implemented all the functions using the same HLS toolchain forcing, where possible, both MaxCompiler and Vivado to perform the same optimisations. Comparing different HLS toolchains, manual VHDL implementations or existing results leads in an invalid comparison as different pipelining choices and scheduling strategies influence both performance and resource utilisation. To the best of our knowledge, there are no available tablebased methods implemented using MaxCompiler that we could use in our experiments. We targeted Xilinx's Alveo U200 Data Center accelerator card. The Alveo U200 incorporates a VU9P FPGA device, with a capacity of 1,182,240 LUTs, 2,364,480 FFs, 6,840 DSPs, 4,320 BRAMs and 960 URAMs. Different data-types and configurations were evaluated: single and double precision floating-point, fixed-point with 4,13 and 4,23 integer and fractional bits respectively. Moreover, for floating-point implementations, the Xilinx FPO operator [34] and in the case of fixed-points, the Xilinx multiplier [40] were used. We configured Vivado to use only one memory type, BRAMs (URAMs are omitted) for a fair comparison.

In the following experiments, we implement each function with a widely used direct method and using Cubic Spline to compare their resource utilisation. Both implementations are pipelined and able to produce (at steady-state) one result per clock cycle. The target frequency was $300 \mathrm{MHz}$ for all hardware implementations. While table-based implementations were able to reach higher clock frequencies compared to direct ones we decided to use the lowest achievable clock frequency by all implementations for the sake of uniformity. Different target clock frequencies will cause MaxCompiler to use more or less aggressive pipelining resulting in an invalid comparison. In all cases, table-based implementations showed lower latency than the direct counterpart even when targeting higher clock frequencies, thus the performance achievable by the table-based implementations is always higher, or at least equal to direct implementations. However, the goal of our methodology is to provide table-based implementations that are equivalent in both performance and accuracy to the direct methods. While worth mentioning, these desirable performance gains are outside the scope of our study and since they could invalidate our experimental results we excluded them from the comparison by using the same configuration in all the experiments. As mentioned earlier we emphasise that the difference in the results from both approaches is smaller than $\epsilon$ by design as they are always computed to the highest precision possible with the given data type. Further details on $\epsilon$ are discussed in section III-B.

The JEF implemented is the Jacobi SN function. The algorithm used for the direct implementation is the common mean method, also known as the Gauss transform method, which is a variant of the descending Landen transformation. The original open-source implementation can be found in "Mathematics Source Library C \& ASM" [41] has been ported without changes on FPGA as a baseline for resource utilisation. The period of the Jacobi SM function can be computed using the complete elliptic integral of the first kind shown in Equation 8. In particular, the Jacobi SN function has a period of $4 K$. For the sake of simplicity, no range reduction was performed and the function was sampled over the entire period. In all the 
tests, the elliptic modulus $k$ is fixed to 0.5 .

$$
K(k)=\int_{0}^{\pi / 2} \sqrt{1-k \sin (t)^{2}} d t
$$

Table I

JACOBI SN FUNCTION RESOURCE UTILISATION

\begin{tabular}{|l|c|c|c|c|c|}
\hline Version & Data-type & LUT & FF & BRAM & DSP \\
\hline Spline & float 11,53 & 7,652 & 12,501 & 13 & 30 \\
\hline Direct & float 11,53 & 751,503 & $1,105,632$ & 713 & 4,522 \\
\hline Spline & float 8,24 & 3,147 & 4,849 & 2 & 8 \\
\hline Direct & float 8,24 & 111,723 & 192,763 & 47 & 1,156 \\
\hline Spline & float 6,10 & 1,580 & 2,237 & 1 & 0 \\
\hline Direct & float 6,10 & 49,720 & 78,783 & 6 & 250 \\
\hline Spline & fixed 4,23 & 387 & 534 & 1 & 3 \\
\hline Direct & fixed 4,23 & 86,593 & 147,419 & 47 & 722 \\
\hline Spline & fixed 4,13 & 387 & 534 & 1 & 3 \\
\hline Direct & fixed 4,13 & 38,610 & 63,564 & 31 & 361 \\
\hline
\end{tabular}

Table I shows that, compared to the direct implementation, the Cubic Spline version saves orders of magnitude of resources in all tests. In the case of double-precision floatingpoint, the LUTs and FFs go from roughly one million needed by the direct implementation to roughly ten thousand used by the Cubic Spline version, achieving a reduction of two orders of magnitude. The DSP utilisation is also reduced by two orders of magnitude with the direct implementation and the Cubic Spline version utilising 4.5k and only 30 DSPs respectively. On the other hand, the BRAMs utilisation is reduced by one order of magnitude from 710 utilised by the direct version to 13 needed by the Cubic Spline version. Similar behaviour can be observed with the remaining data types; the higher the precision required, the more advantageous the Cubic Spline implementation becomes. It is worth noting that in the case of the custom low precision floating-point with 6 exponent bits and 10 mantissa bits (including the implicit 1), no DSPs are needed; The methodology estimates that sampling all the numbers utilises one BRAM at most. Since all possible numbers are sampled there is no need to perform any polynomial interpolation. Further reducing the table size will lead to an underutilised BRAM since it is not possible to consume less than one BRAM block. Such a design will also consume some DSPs for computing the interpolations.

The Legendre polynomial of degree 10 shown in Equation 9 was used for the next test. Since this is a univariate and even function $\left(P_{10}(x)=P_{10}(-x)\right)$, it was sampled on the interval $[0,1)$ instead of $[-1,1)$ since the remaining values can be easily obtained.

$$
\begin{aligned}
P_{10}(x)=\frac{1}{256}( & 46189 x^{10}-109395 x^{8}+ \\
& \left.+90090 x^{6}-30030 x^{4}+3465 x^{2}-63\right)
\end{aligned}
$$

Table II shows that a direct implementation of the Legendre polynomial of degree 10 does not need any BRAM for scheduling. This is expected since the number of multiplications is even, which results in a fully balanced dataflow graph. The only scheduling resources utilised by this implementation
Table II

LEGENDRE POLYNOMIAL DEGREE 10 RESOURCE UTILISATION

\begin{tabular}{|c|c|c|c|c|c|}
\hline Version & Data-type & LUT & FF & BRAM & DSP \\
\hline Spline & float 11,53 & 4,276 & 7,828 & 13 & 30 \\
\hline Direct & float 11,53 & 6,000 & 11,363 & 0 & 113 \\
\hline Spline & float 8,24 & 2,372 & 3,830 & 2 & 8 \\
\hline Direct & float 8,24 & 2,123 & 3,829 & 0 & 38 \\
\hline Spline & float 6,10 & 1,366 & 1,958 & 1 & 0 \\
\hline Direct & float 6,10 & 1,500 & 2,355 & 0 & 14 \\
\hline Spline & fixed 4,23 & 97 & 216 & 1 & 3 \\
\hline Direct & fixed 4,23 & 288 & 1,004 & 0 & 18 \\
\hline Spline & fixed 4,13 & 61 & 132 & 1 & 3 \\
\hline Direct & fixed 4,13 & 134 & 368 & 0 & 9 \\
\hline
\end{tabular}

consist of a few registers required for pipelining. The Cubic Spline implementation offers a trade-off since it utilises some BRAMs to save LUT, FF and DSPs. Compared to the Jacobi $\mathrm{SN}$ function, which gives the best results in resource utilisation, in this case, the resource-saving is not of multiple orders of magnitude except for the DSPs. Given that the two methods produce the same results, with no precision loss, depending on the resource available the user can decide which version is more suitable. For example, Table II shows that, when using single precision floating-points, our approach greatly reduces the number of DSPs utilised but slightly increases the LUTs and FFs utilisation and uses 2 BRAM instead of 0 . While this trade-off seems always more favourable, there can be cases in which the number of BRAMs available is extremely limited compared to the other resources, hence the direct approach can in some cases be preferable.

Our final test concerns Planck's law, which is a bivariate function that has two parameters $\lambda$ and $T$. Since the methodology proposed in this study allows to approximate only univariate functions, Equation 4 was divided into three functions $f(x), g(\lambda, T)$ and $h(y, T)$. Here, $g(\lambda, T)$ scales the input and combines the two arguments $\lambda$ and $T$ into the 1-dimensional variable $x, f(x)$ captures the basic nonlinear dependency of the spectral radiance density on wavelength, and $h(y, T)$ scales the output according to the temperature $T$.

$$
B_{\lambda}(\lambda, T)=h(f(g(\lambda, T)), T)
$$

where:

- $g(\lambda, T)=\frac{\lambda k T}{h c}$

- $h(y, T)=2 y \frac{(k T)^{5}}{h^{4} c^{3}}$

- $f(x)=\frac{1}{x^{5}} \frac{1}{e^{1 / x}-1}$

- The remaining symbols are unchanged from Equation 4.

Table III

PLANCK'S LAW RESOURCE UTILISATION

\begin{tabular}{|c|c|c|c|c|c|}
\hline Version & Data-type & LUT & FF & BRAM & DSP \\
\hline Spline & float 11,53 & 1,254 & 2,288 & 3 & 20 \\
\hline Direct & float 11,53 & 14,166 & 26,205 & 3 & 72 \\
\hline Spline & float 8,24 & 775 & 1,311 & 3 & 10 \\
\hline Direct & float 8,24 & 4,301 & 7,265 & 1 & 19 \\
\hline Spline & float 6,10 & 531 & 944 & 3 & 8 \\
\hline Direct & float 6,10 & 1,500 & 2,355 & 0 & 14 \\
\hline
\end{tabular}


The goal of this experiment is to measure the impact of spline in a complete, if small, numerical physics computation. Table III shows that in all cases the spline implementation consumes far fewer resources than the corresponding direct version. In particular, it can halve the DSP utilisation and reduce by more than $90 \%$ the LUTs and FFs needed at the expense of the same or a couple more BRAMs utilised. Given that $B_{\lambda}(\lambda, T)$ values range from 0 to $10^{13}$ fixed-point data types would use more resources than custom floating-point and were not considered. It is worth noting that further resource-reduction is achievable by value profiling this function and using fixed-point for the Cubic Spline interpolation. Since this optimisation it is not automated in the proposed methodology, it was not performed.

\section{CONCLUSION AND FUtURE WORK}

Our experimental results show that, in some cases, the proposed methodology can lower resource utilisation by orders of magnitude compared to a direct implementation without any performance or accuracy loss. In other cases, the methodology can offer a trade-off in which logic resources can be traded for memory resources. Depending on the resources available on the targeted FPGA device or relevant applications requirements, the developer can select the most suitable implementation. In particular, our approach becomes more advantageous the more the required precision increases. In the case of complex functions whose direct implementation requires a large number of resources, like the JEFs shown in Table I, our table-based polynomial interpolation can save resources up to two orders of magnitude. In the case of a simpler function, for example, the Legendre polynomial shown in Table II, our approach can greatly reduce the DSPs utilisation at the expense of BRAMs. Finally, in the case of a physics application consisting of evaluating Planck's law, Table III shows that our approach can greatly reduce the required LUTs, FFs and DSPs without increasing the BRAMs utilisation.

It is worth mentioning that, given the Data-Flow abstraction provided by MaxCompiler, the architecture represented using such abstraction is throughput-optimised, therefore, pipelined to reach a higher clock frequency and, at steady-state, provide a result at every clock cycle. A reduction in resource utilisation directly translates into a smaller Data-Flow graph consisting of fewer pipeline stages, hence, consistently in all our experiments, our methodology also reduces the latency of the operators. The reduction in resource utilisation can be also translated to higher performance as it would be possible to increase the parallelism by replicating the operator. However, we stress that achieving lower latency is outside the scope of our study, which aims to provide a table based approach equivalent in terms of performance and accuracy, and it might be a notportable optimisation performed by MaxCompiler scheduling algorithm thus not applicable to other HLS toolchains.

As future work, we plan to extend our proposal to support arbitrary intervals $I$ instead of only $[0,1)$. We would also like to apply this methodology to Monte Carlo based simulations, for example [42], or more involved simulations to measure the impact of the proposed method when implementing scientific computing workloads on FPGA. Given the abovestated limitations of our proposed methodology, we plan on implementing a more involved interpolation method based on minimax computed by using the Remez algorithm [31]. This interpolator is inherently more accurate than Cubic Spline and does not require sampling the derivative so, compared to Cubic Splines, it should be at least twice as area-efficient and can also offer an additional resource consumption trade-off as it allows to choose the degree of the polynomial interpolation.

\section{ACKNOWLEDGEMENTS}

This research project was supported by the CRUK Convergence Science Centre at The Institute of Cancer Research, London, and Imperial College London (A26234). We acknowledge funding from the Cancer Research UK programme grant C33589/A19727. The Institute of Cancer Research and The Royal Marsden NHS Foundation Trust are members of the Elekta MR-Linac Research Consortium. We also acknowledge Maxeler Technologies for providing access to their tools, the support, and the expertise contributed to this project.

\section{REFERENCES}

[1] F. Callaly, D. O'Loughlin, D. Lyons, A. Coffey, and F. Morgan, "Xilinx Vivado High Level Synthesis: Case studies," in 25th IET Irish Signals \& Systems Conference 2014 and 2014 ChinaIreland International Conference on Information and Communities Technologies (ISSC 2014/CIICT 2014). Institution of Engineering and Technology, 2014, pp. 352-356. [Online]. Available: https: //digital-library.theiet.org/content/conferences/10.1049/cp.2014.0713

[2] O. Pell, O. Mencer, K. H. Tsoi, and W. Luk, "Maximum Performance Computing with Dataflow Engines," in High-Performance Computing Using FPGAs. New York, NY: Springer New York, 2013, pp. 747-774. [Online]. Available: http://link.springer.com/10. 1007/978-1-4614-1791-0_25

[3] K. Shagrithaya, K. Kepa, and P. Athanas, "Enabling development of OpenCL applications on FPGA platforms," in 2013 IEEE 24th International Conference on Application-Specific Systems, Architectures and Processors. IEEE, 6 2013, pp. 26-30. [Online]. Available: http://ieeexplore.ieee.org/document/6567546/

[4] "XLS: Accelerated HW Synthesis." [Online]. Available: https://google. github.io/xls/

[5] S. P. Summers, "Application of FPGAs to Triggering in High Energy Physics," 2018. [Online]. Available: http://cds.cern.ch/record/2647951? $\ln =$ it

[6] J. Detrey and F. de Dinechin, "Table-based polynomials for fast hardware function evaluation," in Proceedings of the International Conference on Application-Specific Systems, Architectures and Processors, 2005, pp. 328-333.

[7] J.-M. Muller, Elementary Functions. Boston, MA: Birkhäuser Boston, 2016. [Online]. Available: http://link.springer.com/10.1007/ 978-1-4899-7983-4

[8] D. U. Lee, R. C. Cheung, W. Luk, and J. D. Villasenor, "Hardware implementation trade-offs of polynomial approximations and interpolations," IEEE Transactions on Computers, vol. 57, no. 5, pp. 686-701, 2008.

[9] H. C. Neto and M. P. Véstias, "Very low resource table-based FPGA evaluation of elementary functions," in 2013 International Conference on Reconfigurable Computing and FPGAs, ReConFig 2013. IEEE Computer Society, 2013.

[10] S. Nagayama, T. Sasao, and J. T. Butler, "Design method for numerical function generators based on polynomial approximation for FPGA implementation," in Proceedings - 10th Euromicro Conference on Digital System Design Architectures, Methods and Tools, DSD 2007, 2007, pp. 280-287. 
[11] F. De Dinechin, M. Joldes, and B. Pasca, "Automatic generation of polynomial-based hardware architectures for function evaluation," in Proceedings of the International Conference on Application-Specific Systems, Architectures and Processors, 2010, pp. 216-222.

[12] D. B. Thomas, "A General-Purpose Method for Faithfully Rounded Floating-Point Function Approximation in FPGAs," in Proceedings Symposium on Computer Arithmetic, vol. 2015-August. Institute of Electrical and Electronics Engineers Inc., 8 2015, pp. 42-49.

[13] F. De Dinechin and B. Pasca, "Designing custom arithmetic data paths with FloPoCo," IEEE Design and Test of Computers, vol. 28, no. 4, pp. 18-27, 72011.

[14] E. W. Weisstein, "Jacobi Elliptic Functions - from Wolfram MathWorld." [Online]. Available: https://mathworld.wolfram.com/ JacobiEllipticFunctions.html

[15] F. Bowman, Introduction to elliptic functions: With applications. Dover Publications, 1961.

[16] S. Liu, Z. Fu, S. Liu, and Q. Zhao, "Jacobi elliptic function expansion method and periodic wave solutions of nonlinear wave equations," Physics Letters, Section A: General, Atomic and Solid State Physics, vol. 289, no. 1-2, pp. 69-74, 102001.

[17] P. Erdös, "Spiraling the Earth with C. G. J. Jacobi," American Journal of Physics, vol. 68, no. 10, pp. 888-895, 10 2000. [Online]. Available: http://aapt.scitation.org/doi/10.1119/1.1285882

[18] W. Kinnersley, "Exact large amplitude capillary waves on sheets of fluid," Journal of Fluid Mechanics, vol. 77, no. 2, pp. 229-241, 1976. [Online]. Available: https://www.cambridge.org/core/journals/journal-of-fluid-mechanics/ article/abs/exact-large-amplitude-capillary-waves-on-sheets-of-fluid/ 1D4BE6F51EF2555838567193A95571F6

[19] T. E. Baker and A. Bill, "Jacobi elliptic functions and the complete solution to the bead on the hoop problem," American Journal of Physics, vol. 80, no. 6, pp. 506-514, 6 2012. [Online]. Available: http://aapt.scitation.org/doi/10.1119/1.3682321

[20] E. W. Weisstein, "Legendre Polynomial - from Wolfram MathWorld." [Online]. Available: https://mathworld.wolfram.com/ LegendrePolynomial.html

[21] G. Dattoli, "Generalized polynomials, operational identities and their applications," Journal of Computational and Applied Mathematics, vol. 118, no. 1-2, pp. 111-123, 62000.

[22] J. D. Jackson, Classical electrodynamics; 3rd ed. New York, NY: Wiley, 1999. [Online]. Available: https://www.wiley.com/en-gb/ Classical+Electrodynamics\%2C+3rd+Edition-p-9780471309321

[23] A. R. Voelker, I. K. Kaji 'c, and C. Eliasmith, "Legendre Memory Units: Continuous-Time Representation in Recurrent Neural Networks," Tech. Rep., 2019. [Online]. Available: https://github.com/abr/neurips2019

[24] R. Loudon, The quantum theory of light. OUP Oxford, 2000.

[25] M. Planck, "Ueber das Gesetz der Energieverteilung im Normalspectrum," Annalen der Physik, vol. 309, no. 3, pp. 553-563, 1 1901. [Online]. Available: https://onlinelibrary.wiley.com/doi/full/10. 1002/andp.19013090310

[26] N. Zettili, Quantum Mechanics: Concepts and Applications; 2nd ed. New York, NY: Wiley, 2009. [Online]. Available: https://www.wiley.com/en-se/Quantum+Mechanics:+Concepts+ and+Applications, +2nd+Edition-p-9780470026793

[27] G. B. Rybicki and A. P. Lightman, Radiative Processes in Astrophysics, ser. A Wiley-Interscience publication. Wiley, 1991. [Online]. Available: https://books.google.co.uk/books?id=LtdEjNABMlsC
[28] C. Ericson, "Chapter 11 - Numerical Robustness," in Real-Time Collision Detection, ser. The Morgan Kaufmann Series in Interactive 3D Technology, C. Ericson, Ed. San Francisco: Morgan Kaufmann, 2005, pp. 427-463. [Online]. Available: https://www.sciencedirect.com/ science/article/pii/B9781558607323500164

[29] C. de Boor, A Practical Guide to Splines, ser. Applied Mathematical Sciences. New York, NY: Springer New York, 1978, vol. 27. [Online] Available: http://link.springer.com/10.1007/978-1-4612-6333-3

[30] W. Gautschi, Numerical analysis.

[31] P. Markstein, IA-64 and elementary functions : speed and precision. Prentice Hall, 2000

[32] J.-P. Berrut and H. D. Mittelmann, "Adaptive point shifts in rational approximation with optimized denominator," Journal of Computational and Applied Mathematics, vol. 164-165, no. 1, pp. 81-92, 3 2004. [Online]. Available: https://linkinghub.elsevier.com/retrieve/pii/ S0377042703004850

[33] IEEE Computer Society. Microprocessor Standards Committee. Institute of Electrical and Electronics Engineers., and IEEE-SA Standards Board., IEEE standard for floating-point arithmetic. Institute of Electrical and Electronics Engineers, 2008. [Online]. Available: https://ieeexplore.ieee.org/document/4610935

[34] "Xilinx Floating Point Operator." [Online]. Available: https://www. xilinx.com/products/intellectual-property/floating_pt.html

[35] C. A. Hall, "On error bounds for spline interpolation," Journal of Approximation Theory, 1968.

[36] C. A. Hall and W. W. Meyer, "Optimal error bounds for cubic spline interpolation," Journal of Approximation Theory, 1976.

[37] J. Oliver, "Rounding error propagation in polynomial evaluation schemes," Journal of Computational and Applied Mathematics, vol. 5, no. 2, pp. 85-97, 61979.

[38] "Performance and Resource Utilization for Floating-point v7.1." [Online]. Available: https://www.xilinx.com/support/documentation/ip_ documentation/ru/floating-point.html

[39] F. De Dinechin, B. Pasca, O. Cret, and R. Tudoran, "An FPGA-specific approach to floating-point accumulation and sum-of-products," in Proceedings of the 2008 International Conference on Field-Programmable Technology, ICFPT 2008, 2008, pp. 33-40.

[40] "Xilinx Multiplier." [Online]. Available: https://www.xilinx.com/ products/intellectual-property/multiplier.html

[41] "Elliptic Functions." [Online]. Available: http://www.mymathlib.com/ functions/elliptic functions.html

[42] N. Voss, P. Ziegenhein, L. Vermond, J. Hoozemans, O. Mencer, U. Oelfke, W. Luk, and G. Gaydadjiev, "Towards real time radiotherapy simulation," in Proceedings of the International Conference on Application-Specific Systems, Architectures and Processors, vol. 2019. July. Institute of Electrical and Electronics Engineers Inc., 7 2019, pp. 173-180. 